than lull their users into a false sense of security. If we are going to recommend the fitting of radar reflectors, we should specify what is and what is not a good reflector.

\title{
The True-motion Radar Display
}

\author{
from Captain R. G. Swallow, R.N.
}

(Radio Advisory Service)

TRUE-MOTION radar will tell you the range, compass bearing and true course of target echoes and give you facilities for estimation or calculation of target speed. It will not, however, give you the closest-approach distance without plotting or without using a closest-approach distance calculator.

If, however, it was possible to set own ship's speed to zero without having to revert to the relative display, the true-motion of echoes would change to relative motion in continuation from their present known position on the display, and using the reflection plotter the relative course of an echo could very quickly be observed and extended past own ship's position to show the closest approach distance. Setting own ship's speed back to its correct figure would then put echoes back to true-motion presentation and it would be quickly apparent if any of them had in the interval altered from their original true course.

An unsolved problem of this display is the time lag before alterations of course or speed by a target echo can be definitely appreciated from the movement of echoes on the PPI. The time lag is reduced on the true-motion display but nevertheless some delay still remains.

Finally it must not be forgotten that when true-motion presentation is used, a major part of the problem of manœuvring action for collision avoidance still remains, because, although the course steered by the other vessel may be seen, it is just as difficult to know how to proceed in order to keep her outside a certain safe distance. Before making a decision the situation must be analysed and, as there are no steering rules other than Rules 25 and 27 applicable to ships not in sight of one another, one must reflect before acting.

\section{from J. Th. Verstelle}

\section{(Royal Netherlands Hydrographic Office)}

TRUE-MOTION radar seems to me a most valuable, simple and elegant solution of the problems of relative motion. Where many ships are involved there is simply no time for the usual procedure of plotting. The conclusion might have been drawn from some of the papers that only in true-motion radar do errors in the assumed course and speed falsify the conclusions. In any particular case, however, the same errors would have been introduced into the normal plot (and into the results from the reflection plotter) and would influence the conclusions in exactly the same way and by exactly the same amount. 